\title{
DETERMINAÇÃO DE MATÉRIA ORGÂNICA DO SOLO PELA PERDA DE MASSA POR IGNIÇÃO, EM AMOSTRAS DO RIO GRANDE DO SUL ${ }^{(1)}$
}

\author{
Pedro Alexandre Varella Escosteguy ${ }^{(2)}$, \\ Katiusca Galliassi $^{(3)}$ \& Carlos Alberto Ceretta ${ }^{(4)}$
}

\begin{abstract}
RESUMO
A busca de métodos que possibilitam a determinação do teor de matéria orgânica (MO) do solo e que não geram resíduos danosos ao ambiente é importante. A oxidação da MO com dicromato é amplamente utilizada no Brasil, gerando resíduos laboratoriais que contêm $\mathrm{Cr}$. Este trabalho teve por objetivo avaliar o método da perda de massa por ignição (PMI), em comparação ao método da combustão úmida usando a solução sulfocrômica, utilizado na Rede Oficial de Laboratórios de Análise de Solo e de Tecido Vegetal dos Estados do Rio Grande do Sul e de Santa Catarina (ROLAS). Os resultados obtidos com a PMI também foram comparados com os do método-padrão de Walkley-Black, que também emprega o dicromato. Foram utilizados os resultados de amostras de solo de 19 unidades de mapeamento do Rio Grande do Sul (RS) e de 49 amostras da Depressão Central deste Estado. As 19 amostras incluíram: Vertissolo (1), Gleissolos (2), Argissolos (6), Luvissolo (1), Neossolo (1), Latossolos (3), Plintossolo (1), Planossolo (1), Alissolo (1), Nitossolo (1) e Chernossolo (1). As amostras da Depressão Central foram obtidas no Laboratório de Solos da Universidade Federal de Santa Maria. Os teores de MO determinados com a PMI foram, em média, 22 a 94 \% maiores que os da solução sulfocrômica e 27 a 46 \% maiores que os obtidos com o Walkley-Black, nas amostras da Depressão Central e nas unidades de mapeamento do RS, respectivamente. Houve correlação significativa entre a PMI e os métodos com dicromato. Os resultados indicam que o método PMI pode ser usado na análise do teor de MO do solo em substituição aos que empregam o dicromato. Para isso, equações que possibilitam calcular o teor de MO usando a PMI devem ser desenvolvidas para a maioria das classes de solos do RS.
\end{abstract}

Termos de indexação: cromo, análise de solo, carbono orgânico, contaminação.

(1) Financiado pela Secretaria de Ciência e Tecnologia do Rio Grande do Sul. Apresentado na XXXV Reunião Anual da Rede Oficial de Laboratórios de Análise de Solo e de Tecido Vegetal dos Estados do Rio Grande do Sul e de Santa Catarina (ROLAS). Recebido para publicação em abril de 2006 e aprovado em janeiro de 2007.

${ }^{(2)}$ Professor da Faculdade de Agronomia e Medicina Veterinária, Universidade de Passo Fundo - UPF. Caixa Postal 611, CEP 99001-970 Passo Fundo (RS). E-mail: escosteguy@upf.br.

(3) Acadêmica do Curso de Química, UPF.

(4) Professor do Departamento de Solos, Universidade Federal de Santa Maria - UFSM. Santa Maria (RS). Campus Universitário, CEP 97105-900 Santa Maria (RS). E: mail: ceretta@ccr.ufsm.br 


\title{
SUMMARY: SOIL ORGANIC MATTER DETERMINATION BY WEIGHT LOSS- ON-IGNITION IN SAMPLES FROM THE STATE OF RIO GRANDE DO SUL, BRAZIL
}

\begin{abstract}
The search for methods to determine the content of soil organic matter (SOM) that do not generate hazardous waste is important. SOM oxidation with dichromate is used in most Brazilian soil laboratories and it generates chromium-contaminated waste. The objective of this study was to evaluate the weight loss-on-ignition (LOI) method in comparison to the wet digestion procedure based on sulfuric acid-chromium solution as commonly used in soil analysis laboratories of Rio Grande do Sul (RS) and Santa Catarina (SC) states, Brazil. The results obtained by the LOI method were further compared with the Walkley-Black standard-method, which also uses dichromate. The SOM content was determined in 19 samples from different soil units in $R S$ and in 49 samples from the Central Depression of the same state. The 19 samples included: Vertisol (1), Gleysols (2), Argisols (6), Luvisol (1), Neosol (1), Latosols (3), Plinthosol (1), Planosol (1), Alisol (1), Nitosol (1), and Mollisol (1). Central Depression samples were provided by the Federal University of Santa Maria Soil Analysis Laboratory. The mean SOM contents obtained by LOI were 22 and $94 \%$ higher than the means obtained with the sulfuric acid-chromium solution, and 27 and $46 \%$ higher than that obtained with the Walkley-Black method, in Central-Depression and other $R S$ soil samples, respectively. The LOI method was significantly correlated with the dichromate methodologies. Results indicate LOI for SOM analysis as a replacement of the dichromate methods. Equations that estimate the SOM content based on LOI should be developed for all RS soil classes.
\end{abstract}

Index terms: Chromium, soil test, organic carbon, environmental contamination.

\section{INTRODUÇÃO}

Nos laboratórios integrantes da Rede Oficial de Laboratórios de Análise de Solo e de Tecido Vegetal dos Estados do Rio Grande do Sul e de Santa Catarina (ROLAS), o método da oxidação do C orgânico por dicromato mais ácido sulfúrico, denominado solução sulfocrômica, é utilizado para determinar o teor de MO do solo em análises básicas (Sociedade..., 2004). Embora esse método tenha boa exatidão e seja considerado simples e de baixo custo (Tedesco et al., 1995), a utilização do dicromato resulta na adição de $\mathrm{Cr}$ aos resíduos líquidos. Esse metal ocorre naturalmente no ambiente no estado de oxidação trivalente $\left(\mathrm{Cr}^{3+}\right)$, sendo considerado um elemento essencial ou benéfico aos seres vivos. Entretanto, quando na forma hexavalente $\left(\mathrm{Cr}^{6+}\right)$, é considerado tóxico aos seres humanos, pois, em contato com a pele, ou via ingestão, pode causar ulcerações, irritação e inflamação e está associado a risco de câncer (Comissão..., 2004).

Como na maioria dos laboratórios os resíduos líquidos não são tratados antes do descarte no ambiente, a utilização da solução sulfocrômica para determinação da MO do solo preocupa, pois esse método pode ser uma fonte de disseminação de Cr. Embora o $\mathrm{Cr}^{6+}$ nos resíduos laboratoriais possa ser neutralizado, por exemplo, com processos de redução e precipitação, isso não evita que seja gerado um resíduo sólido que contém esse metal. De acordo com a NBR 10.004, da Associação Brasileira de Normas Técnicas, esses resíduos são classificados como perigosos, ou Classe I
(ABNT, 2004). Tais resíduos necessitam de um sistema especial de acondicionamento, transporte, tratamento e, ou, disposição, o que dificulta o emprego de processos de tratamento nos laboratórios. Essa dificuldade é acentuada no caso dos laboratórios localizados no interior do RS, onde são raros os aterros de resíduos industriais perigosos.

Os métodos de extração do C orgânico do solo são agrupados da seguinte maneira por Nelson \& Sommers (1996): (1) diferença entre o C total e o C inorgânico, (2) determinação do $\mathrm{C}$ total, após a remoção do C inorgânico, (3) oxidação por dicromato sem ou (4) com uma fonte externa de aquecimento. Contudo, o método de combustão seca é considerado mais exato para determinar esse atributo do solo (Kimble et al., 2001). A aplicação desses métodos varia com o propósito da análise de solo. Em laboratórios de rotina no Brasil, o método do dicromato é o mais utilizado, pois é de simples execução e dispensa o uso de equipamentos especializados, além de apresentar boa exatidão e oxidar as frações de MO mais reativas no solo (Tedesco et al., 1995). As limitações do método com dicromato têm sido, tradicionalmente, relacionadas com interferências, devido à oxidação ou redução de alguns constituintes do solo, como o $\mathrm{Cl}^{-}$,

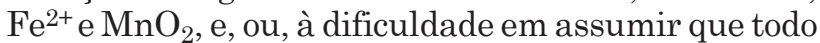
o C orgânico na amostra possui valência zero (Nelson \& Sommers, 1996). Outra limitação desse método tem sido relacionada ao impacto ambiental do Cr. Esse aspecto motivou, em outros países, a substituição do método com dicromato pelo método perda de massa por ignição (PMI) (Combs \& Nathan, 1998; 
Cambardella et al., 2001; Konen et al., 2002). O método PMI consiste na determinação gravimétrica do $\mathrm{C}$ transformado em $\mathrm{CO}_{2}$, o qual é estimado pela massa volatilizada de uma amostra de solo seco submetido à ignição em alta temperatura (Schulte \& Hopkins, 1996).

Até recentemente, o método PMI não era utilizado em laboratórios de rotina, principalmente devido ao prolongado tempo para a pesagem das amostras. Entretanto, nos últimos anos, a maior preocupação com a qualidade do ambiente nos países desenvolvidos estimulou o uso desse método, o qual foi viabilizado com a automatização dos procedimentos de pesagem. A adoção do método PMI também é limitada pela necessidade de os resultados obtidos com ele serem correlacionados com os métodos usados em tabelas com recomendação de adubação nitrogenada, e essa correlação pode variar com o tipo e o uso do solo (Combs \& Nathan, 1998).

Este trabalho teve como objetivo avaliar a possibilidade de utilização do método PMI para determinação dos teores de MO do solo, em substituição ao método que emprega a solução sulfocrômica.

\section{MATERIAL E MÉTODOS}

Dois conjuntos de amostras de solo foram analisados, em triplicatas. No primeiro conjunto foram incluídas 19 amostras, coletadas na profundidade de $0-10 \mathrm{~cm}$, representando diversas unidades de mapeamento do Estado do Rio Grande do Sul (Quadro 1). Após a coleta, essas amostras foram secas em estufa $\left(50{ }^{\circ} \mathrm{C}, 48 \mathrm{~h}\right)$, moídas e peneiradas (abertura de malha de $2 \mathrm{~mm}$ ). Os teores de MO dessas amostras foram determinados com os métodos PMI, Walkley-Black com aquecimento externo e solução sulfocrômica. Os resultados obtidos com o método PMI foram correlacionados com os observados em outros métodos. Posteriormente, esses resultados foram reunidos com os obtidos em 49 amostras de solos da Região Fisiográfica da Depressão Central do RS, as quais foram analisadas por Escosteguy et al. (2002), com o propósito de melhor representar os solos desse Estado. Formou-se, então, um segundo conjunto de dados, constituído pelos resultados das 19 amostras do primeiro conjunto mais os obtidos nas 49 amostras da Depressão Central. Esse conjunto de dados foi submetido à análise de regressão, para, novamente, correlacionar os resultados obtidos pelo método PMI com os dos métodos que utilizam o dicromato.

$\mathrm{Na}$ determinação do teor de $\mathrm{C}$ com o método PMI utilizaram-se latas inox $\left(24 \mathrm{~cm}^{3}\right)$, sendo as pesagens efetuadas em balança analítica (quatro dígitos decimais). As latas foram aquecidas em estufa $\left(360 \pm 2{ }^{\circ} \mathrm{C}, 2 \mathrm{~h}\right)$ com circulação de ar e deixadas esfriar para pesagem. Após a pesagem das latas (tara), pesaram-se as amostras de solo $\left(1,5 \mathrm{~cm}^{3}\right)$. A seguir, essas amostras foram secadas em estufa

Quadro 1. Classificação taxonômica e teores de argila e de carbono orgânico (C) das amostras de solos das diversas unidades de mapeamento do Rio Grande do Sul

\begin{tabular}{|c|c|c|c|c|}
\hline Amostra & Unidade de mapeamento & Classificação & Argila & $\mathbf{C}^{(1)}$ \\
\hline & & & $\mathrm{g} \mathrm{kg}^{-1}$ & $\mathrm{~g} \mathrm{dm}{ }^{-3}$ \\
\hline 1 & Banhado & Gleissolo Háplico Ta eutrófico vértico & 449 & 12,6 \\
\hline 2 & Bom Retiro & Argissolo Vermelho distrófico arênico & 149 & 4,5 \\
\hline 3 & Charrua & Neossolo Litólico eutrófico chernossólico & 389 & 27,4 \\
\hline 4 & Colégio & Gleissolo Melânico eutrófico típico & 89 & 9,0 \\
\hline 5 & Cruz Alta & Latossolo Vermelho distrófico típico & 189 & 10,1 \\
\hline 6 & Durasnal & Plintossolo Argilúvico eutrófico abrúptico & 269 & 11,1 \\
\hline 7 & Escobar & Vertissolo Ebânico órtico típico & 409 & 22,9 \\
\hline 8 & Estação & Latossolo Vermelho distroférrico nitossólico & 729 & 17,1 \\
\hline 9 & Itapoã & Argissolo Vermelho-Amarelo distrófico arênico & 129 & 3,8 \\
\hline 10 & Júlio de Castilho & Argissolo Vermelho -Amarelo alumínico típico & 436 & 13,5 \\
\hline 11 & Pelotas & Planossolo Hidromórfico eutrófico solódico & 189 & 9,6 \\
\hline 12 & Santa Maria & Alissolo Crômico argilúvico típico & 161 & 8,3 \\
\hline 13 & Santo Ângelo & Latossolo Vermelho distroférrico típico & 636 & 11,4 \\
\hline 14 & São Borja & Nitossolo Vermelho distroférrico latossólico & 529 & 10,9 \\
\hline 15 & São Jerônimo & Argissolo Vermelho distrófico típico & 309 & 10,9 \\
\hline 16 & São Pedro & Argissolo Vermelho distrófico arênico & 129 & 7,1 \\
\hline 17 & Tupanciretã & Argissolo Vermelho-Amarelo distrófico típico & 109 & 2,6 \\
\hline 18 & Vila & Chernossolo Háplico órtico típico & 209 & 15,9 \\
\hline 19 & Virgínia & Luvissolo Crômico pálico abrúptico & 349 & 16,1 \\
\hline
\end{tabular}

(1) Determinado pelo método de Walkley-Black. 
$\left(105 \pm 2{ }^{\circ} \mathrm{C}, 2 \mathrm{~h}\right)$ e, após esfriar, pesadas novamente. $\mathrm{Na}$ seqüência, as amostras foram aquecidas em estufa $\left(360 \pm 2{ }^{\circ} \mathrm{C}, 2 \mathrm{~h}\right)$ e, depois de esfriadas, pesadas novamente. $\mathrm{O}$ teor $(\% \mathrm{p} / \mathrm{p})$ de $\mathrm{MO}$ foi calculado com a fórmula:

100 (peso da lata + peso solo $105^{\circ} \mathrm{C}$ ) - (peso da lata + peso solo $360^{\circ} \mathrm{C}$ )

(peso da lata + peso solo $105^{\circ} \mathrm{C}$ ) - (peso da la ta)

Para análise da MO com o método Walkley-Black, utilizou-se o procedimento descrito em Nelson \& Sommers (1996). Os resultados foram expressos em teores de MO, assumindo 58 \% de C na composição da MO do solo (fator de Van Bemmelen = 1,72).

A determinação dos teores de $\mathrm{MO}$ com o método da solução sulfocrômica foi efetuada nos Laboratórios de Solos da Universidade Federal de Santa Maria (amostras da Depressão Central) e da Universidade de Passo Fundo (demais amostras). O procedimento utilizado nesses laboratórios foi o descrito em Tedesco et al. (1995).

A determinação dos modelos de regressão linear simples, a análise da variância da equação de regressão, o teste $t$ do coeficiente angular e o teste de falta de ajuste do modelo foram efetuados utilizando o programa Minitab (versão 10.2).

\section{RESULTADOS E DISCUSSÃO}

\section{Amostras de diversas unidades de mapeamento}

Os resultados obtidos com as análises das amostras coletadas em diversas unidades de mapeamento do $\mathrm{RS}$ mostram que houve relação entre os teores de MO determinados com o método PMI e os obtidos com o Walkley-Black, sendo essa relação descrita pelo modelo linear $\mathrm{MO}_{\mathrm{WB}}=0,379+0,543 \mathrm{MO}_{\mathrm{PMI}}$ (Figura 1a; Quadro 2). Os resultados obtidos na amostra da unidade de mapeamento Escobar foram desconsiderados, pois prejudicaram o ajuste desse modelo. O resíduo-padrão dos resultados obtidos na análise de regressão desta amostra foi maior do que 2, o que não ocorreu com as demais amostras (resultados não apresentados). O teor de MO na amostra da unidade de mapeamento Escobar, com o método PMI, foi maior do que o observado nas demais amostras. Isso, possivelmente, deve-se à presença de montmorilonita no horizonte superficial desse solo (Brasil, 1973). Quando rico em Na, esse silicato perde água da estrutura mineralógica entre 150 e $250^{\circ} \mathrm{C} \mathrm{e}$, quando rico em $\mathrm{Ca}$, isso ocorre entre 250 e $350^{\circ} \mathrm{C}$ (Schulte \& Hopkins, 1996). Assim, a água estrutural desse silicato deve ter evaporada quando do aquecimento da amostra a $360{ }^{\circ} \mathrm{C}$, e a massa correspondente a essa água foi considerada como sendo de compostos orgânicos, na análise feita com o método PMI.

Houve relação entre os teores de MO determinados com o método PMI e os obtidos com a solução sulfocrômica, sendo essa relação descrita pelo modelo linear $\mathrm{MO}_{\text {ROLAS }}=0,483+0,316 \mathrm{MO}_{\mathrm{PMI}}$ (Figura 1b; Quadro 2). Isso era esperado, pois a solução sulfocrômica estima de forma satisfatória os teores de MO determinados com o método Walkley-Black (Tedesco et al., 1995), e os resultados obtidos com esse último método tiveram relação significativa com os determinados com o método PMI (Figura 1a).
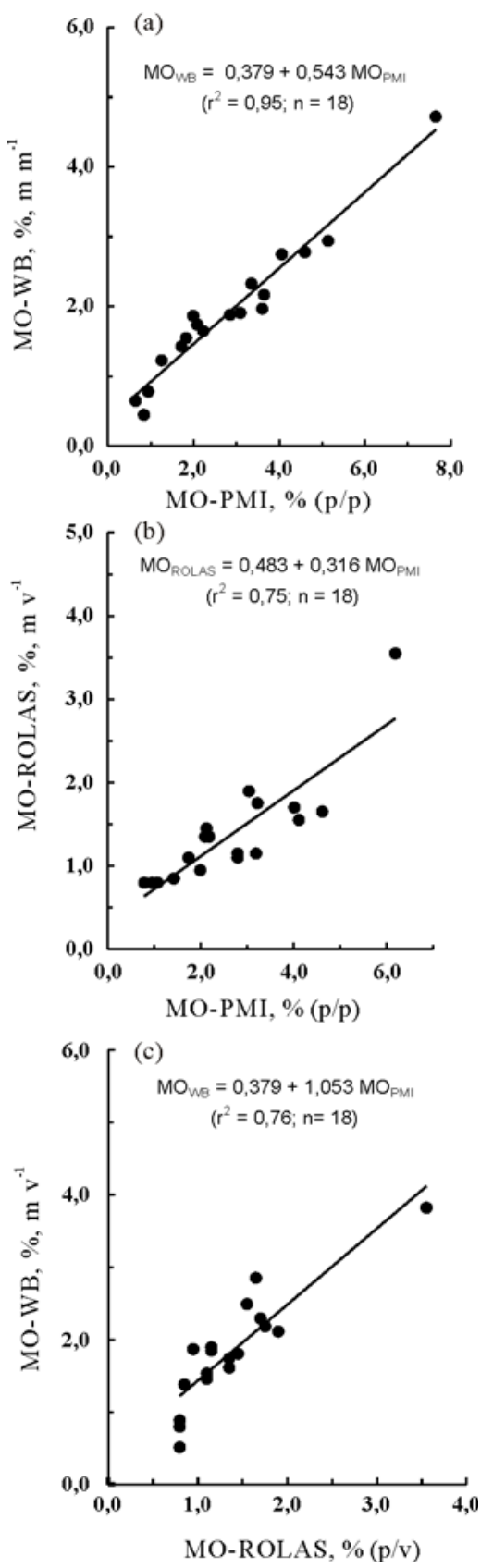

Figura 1. Relação entre os teores de matéria orgânica (MO) determinada com os métodos WalkleyBlack (WB), perda de massa por ignição (PMI) e solução sulfocrômica (ROLAS), em amostras de solos de diversas unidades de mapeamento do Rio Grande do Sul. 
$\mathrm{O}$ valor do coeficiente de determinação da equação de regressão obtida entre os métodos Walkley-Black e PMI ( $r^{2}=0,95$; Figura 1a) foi maior do que o observado na regressão entre esse método e a solução sulfocrômica $\left(r^{2}=0,75\right.$; Figura $\left.1 b\right)$. Isso também foi verificado em outros trabalhos que compararam esses três métodos utilizando amostras dos Estados Unidos e da região de Passo Fundo, RS (Escosteguy et al., 2002) e amostras do Estado de Santa Catarina (Escosteguy et al., 2003). Esses podem estar relacionados às diferenças intrínsecas entre esses métodos ou ao fato de as análises efetuadas com a solução sulfocrômica terem sido realizadas por laboratório de rotina, enquanto as análises com o Walkley-Black foram efetuadas em laboratório de pesquisa.

$\mathrm{O}$ valor do $\mathrm{r}^{2}$ da equação de regressão obtida entre os métodos Walkley-Black e PMI $\left(\mathrm{r}^{2}=0,95\right.$; Figura 1a) também foi maior do que o obtido na regressão entre os métodos Walkley-Black e solução sulfocrômica $\left(r^{2}=0,76\right.$; Figura 1c). Como o primeiro é métodoreferência em calibrações de métodos (Tedesco et al., 1995), isso indica que, de maneira geral, o método PMI estimou de forma adequada os teores de MO desse conjunto de amostras.

\section{Amostras de diversas unidades de mapeamento reunidas com as da Depressão Central}

Para abranger maior número de tipos de solos e incluir amostras com teores de MO maiores do que 4,0 \%, obtidos com o método utilizado pela ROLAS, o que não ocorreu nas amostras coletadas em diversas unidades de mapeamento do RS (Figura 1), os resultados dessas amostras foram reunidos com os obtidos na análise das amostras de solos da Região Fisiográfica da Depressão Central, e os métodos estudados foram relacionados.
Como mostram os resultados (Figura 2a e Quadro 2), houve relação linear entre os teores de MO determinados com os métodos PMI e Walkley-Black $\left(\mathrm{MO}_{\mathrm{WB}}=0,063+0,765 \mathrm{MO}_{\mathrm{PMI}}\right)$. Os valores dos coeficientes dessa equação $(a=0,063$ e $b=0,765)$ foram diferentes dos obtidos com a análise de regressão dos resultados do primeiro conjunto de amostras analisadas ( $a=0,379$ e $b=0,543$; Figura 1a). Embora o valor do $\mathrm{r}^{2}$ tenha decrescido com a inclusão das amostras da Depressão Central no conjunto de dados analisados $\left(\mathrm{r}^{2}=0,95\right.$ e 0,84 ; Figuras $1 \mathrm{a}$ e $2 \mathrm{a}$, respectivamente), o valor de 0,84 pode ser considerado razoável.

Como ocorreu no primeiro conjunto de dados analisado, o modelo linear também descreveu de forma significativa a relação entre os resultados de MO obtidos com os métodos solução sulfocrômica e PMI. Essa relação foi descrita pela equação $\mathrm{MO}_{\text {ROLAS }}$ $=0,027+0,805 \mathrm{MO}_{\mathrm{PMI}}$ (Figura 2b; Quadro 2), e os valores dos coeficientes $(a=0,027$ e $b=0,805)$ também foram diferentes dos obtidos com as amostras das diversas unidades de mapeamento do $\mathrm{RS}$ ( $a=0,483$ e $b=0,316$; Figura 1b). Ao comparar 16 equações de regressão linear que relacionam os métodos com dicromato com o método PMI, Schulte \& Hopkins (1996) observaram diferenças entre os valores dos coeficientes dessas equações. Segundo esses autores, isso resulta dos diferentes tempos e temperatura de aquecimento da amostra analisada com o método PMI e, ainda, pode estar relacionado com possíveis diferenças de tipo de argila e frações de MO das amostras analisadas. Esse último aspecto também pode ter influenciado os resultados obtidos neste trabalho, pois há diferenças expressivas de teor de argila e composição mineral entre os solos dos dois conjuntos de amostras analisadas (Brasil, 1973).

Quadro 2. Níveis de significância da análise da variância (Modelo) e dos testes do coeficiente angular (b) e de falta de ajuste dos modelos ajustados com os teores de matéria orgânica (MO), obtidos com os métodos Walkley-Black (WB), perda de massa por ignição (PMI) e solução sulfocrômica (ROLAS), em amostras de solos do Rio Grande do Sul

\begin{tabular}{|c|c|c|c|c|}
\hline \multirow{2}{*}{ Figura } & \multirow{2}{*}{ Equação ajustada } & \multicolumn{3}{|c|}{ Nível de significância } \\
\hline & & Modelo & b & Falta de ajuste \\
\hline \multicolumn{5}{|c|}{ Diversas unidades de mapeamento $(\mathrm{n}=18)$} \\
\hline $1 \mathrm{a}$ & $\mathrm{MO}_{\mathrm{wB}}=0,379+0,543 \mathrm{MO}_{\mathrm{PMI}}$ & $<0,001$ & $<0,001$ & $>0,1$ \\
\hline $1 b$ & $\mathrm{MO}_{\text {ROLAS }}=0,483+0,316 \mathrm{MO}_{\mathrm{PMI}}$ & $<0,001$ & $<0,001$ & 0,074 \\
\hline $1 \mathrm{c}$ & $\mathrm{MO}_{\mathrm{wB}}=0,379+1,053 \mathrm{MO}_{\mathrm{ROLAS}}$ & $<0,001$ & $<0,001$ & $>0,1$ \\
\hline \multicolumn{5}{|c|}{ Diversas unidades de mapeamento e Depressão Central $(\mathrm{n}=65)$} \\
\hline $2 \mathrm{a}$ & $\mathrm{MO}_{\mathrm{WB}}=0,063+0,765 \mathrm{MO}_{\mathrm{PMI}}$ & $<0,001$ & $<0,001$ & $>0,1$ \\
\hline $2 \mathrm{~b}$ & $\mathrm{MO}_{\text {ROLAS }}=0,027+0,805 \mathrm{MO}_{\mathrm{PMI}}$ & $<0,001$ & $<0,001$ & $>0,1$ \\
\hline $2 \mathrm{c}$ & $\mathrm{MO}_{\mathrm{wB}}=0,500+0,764 \mathrm{MO}_{\mathrm{ROLAS}}$ & $<0,001$ & $<0,001$ & $>0,1$ \\
\hline
\end{tabular}


Ao contrário do observado na regressão ajustada entre os métodos Walkley-Black e PMI, o valor do ${ }^{2}$ obtido na regressão entre os métodos solução sulfocrômica e PMI aumentou com o acréscimo do número de amostras $\left(\mathrm{r}^{2}=0,75\right.$ e 0,80; Figuras $1 \mathrm{~b}$ e $2 \mathrm{~b}$, respectivamente). Possivelmente, isso se deve à inclusão das amostras dos solos da Depressão Central nesse segundo conjunto de dados analisados, pois, ao analisar somente as amostras dessa região e correlacionar esses dois métodos, Escosteguy et al.
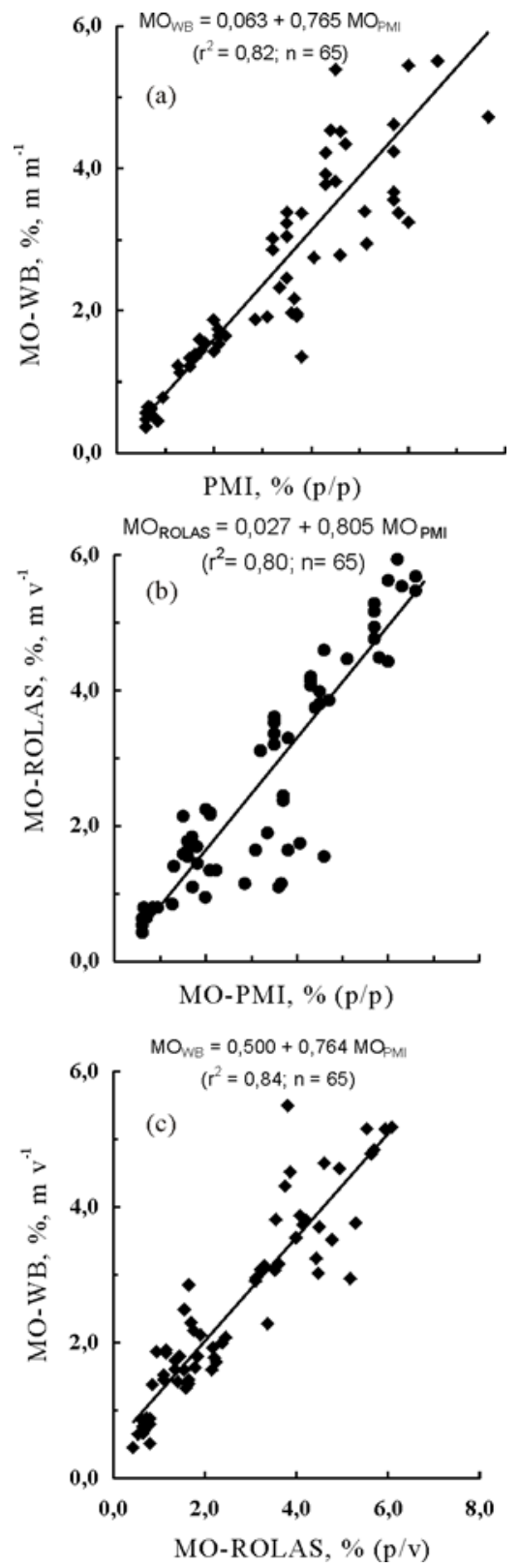

Figura 2. Relação entre os teores de matéria orgânica (MO) determinada com os métodos WalkleyBlack (WB), perda de massa por ignição (PMI) e solução sulfocrômica (ROLAS), em amostras de solos de diversas unidades de mapeamento e da Depressão Central do Rio Grande do Sul.
(2002) obtiveram valor de $\mathrm{r}^{2}=0,98$. Além disso, isso pode ter relação com o fato de as análises realizadas com o método da solução sulfocrômica terem sido efetuadas por diferentes laboratórios de rotina (UPF e UFSM), incluindo, portanto, variabilidade interlaboratorial, ao passo que as análises feitas com o Walkley-Black foram efetuadas em laboratório de pesquisa.

A inclusão das amostras da Depressão Central no conjunto de dados analisados melhorou o $\mathrm{r}^{2}$ obtido entre os métodos Walkley-Black e solução sulfocrômica $\left(\mathrm{r}^{2}=0,76\right.$ e 0,81 ; Figuras $1 \mathrm{c}$ e $2 \mathrm{c}$, respectivamente), sendo o valor desse coeficiente semelhante ao obtido na regressão entre os métodos PMI e Walkley-Black $\left(r^{2}=0,82\right.$; Figura 2a). Como observado no primeiro conjunto de dados, esse grau de ajuste da regressão obtida com os métodos Walkley-Black e PMI indica que este último estima de forma adequada os teores de MO das amostras analisadas, considerando o seu uso em análise de rotina.

A análise estatística das regressões lineares, obtidas com os dois conjuntos de amostras estudadas (Figuras 1 e 2), indicou que os ajustes das equações e dos coeficientes angulares foram altamente significativos $(p<0,001)$ e que não houve falta de ajuste dos modelos (Quadro 2). Como mostram os resultados (Quadro 2), o nível de significância do teste de falta de ajuste do modelo que relaciona os resultados dos métodos PMI e solução sulfocrômica, obtidos com as amostras das diversas unidades de mapeamento do RS, foi de 0,074. Embora esse valor tenha sido pequeno em relação ao dos demais modelos (nível de significância > 0,1; Quadro 2), ele não foi menor do que $5 \%(\mathrm{P}<0,05)$, sendo, portanto, adequado.

Considerando o maior $\mathrm{r}^{2}$ e o maior número de solos representados no modelo obtido no segundo conjunto de dados, é preferível estimar o teor de MO determinado com a solução sulfocrômica usando a equação $\mathrm{MO}_{\text {ROLAS }}=0,027+0,805 \mathrm{MO}_{\mathrm{PMI}}$, ajustada com esse conjunto de dados (Figura 2b), em detrimento da equação $\mathrm{MO}_{\text {ROLAS }}=0,483+0,316 \mathrm{MO}_{\mathrm{PMI}}$, obtida com o primeiro conjunto de amostras analisadas (Figura 1b).

As tabelas utilizadas para recomendação da adubação nitrogenada no Rio Grande do Sul (Sociedade..., 2004) são baseadas nos teores de MO do solo. Esse atributo é determinado com o método da solução sulfocrômica, em laboratório de rotina, sendo os valores obtidos com esse método, portanto, imprescindíveis nos laudos emitidos por esses laboratórios. Assim, se o teor desse atributo for determinado com o método PMI, deve-se, então, estimar o teor de MO que corresponderia ao obtido com a solução sulfocrômica, pois isso possibilitaria que o método PMI fosse utilizado em laboratórios de rotina. Os resultados deste trabalho mostram que não foi possível extrapolar a equação obtida com as amostras das diversas unidades de mapeamento do RS $\left(\mathrm{MO}_{\text {ROLAS }}=0,483+0,316 \mathrm{MO}_{\mathrm{PMI}} ;\right.$ Figura $\left.1 \mathrm{~b}\right)$ com a 
obtida quando essas amostras foram reunidas com as da Depressão Central $\left(\mathrm{MO}_{\text {ROLAS }}=0,027+0,805\right.$ $\mathrm{MO}_{\mathrm{PMI}}$; Figura 2b). Embora os resultados mostrem boa relação entre os métodos PMI e solução sulfocrômica (Figura 2b; Quadro 2), a equação que correlaciona esses dois métodos deve ser validada utilizando maior número de classes de solos representativas do Estado do RS.

\section{Forma de expressão dos resultados obtidos com o método PMI}

Os valores da estimativa da média, da mediana e do valor máximo dos teores obtidos com o método PMI foram, em geral, maiores do que os observados nos métodos solução sulfocrômica e Walkley-Black (Quadro 3). Nas amostras das unidades de mapeamento do RS, o teor médio de MO determinado com a PMI foi de 2,69\%, contra 1,84 e 1,39\%, determinados com o Walkley-Black e a solução sulfocrômica, respectivamente (Quadro 3). Nas amostras da Depressão Central, os teores médios de MO foram de 3,22 \% (PMI), 2,53 \% (Walkley-Black) e $2,65 \%$ (solução sulfocrômica) (Quadro 3). Assim, o método da PMI superestimou os teores de MO determinados com o Walkley-Black e com a solução sulfocrômica, respectivamente, em 94 e $46 \%$ (unidades de mapeamento) e em 22 e 27 \% (Depressão Central). Isso pode estar relacionado com a volatilização de substâncias não-orgânicas, quando a amostra analisada é aquecida a $360{ }^{\circ} \mathrm{C}$ - valor de temperatura usado no método PMI. Além do $\mathrm{C}$ orgânico, água de hidratação e certos compostos minerais também volatilizam nessa faixa de temperatura (Ben-Dor \& Banin, 1989). Esses autores informam que a desidratação de filossilicatos e do gesso ocorre entre 100 e $200{ }^{\circ} \mathrm{C}$, enquanto a da haloisita e dos óxidos de $\mathrm{Fe}$, comuns nas amostras analisadas (Brasil, 1973), acontece entre 200 e $300{ }^{\circ} \mathrm{C}$ e 250 e $300^{\circ} \mathrm{C}$, respectivamente.

Os resultados também indicam que, quando analisados os teores de MO dos dois conjuntos de amostras, os valores máximo e mínimo, da média e da mediana, estimados com os resultados obtidos com o método solução sulfocrômica, superestimaram os obtidos com o Walkley-Black. Entretanto, com o primeiro conjunto de amostras ocorreu o contrário, ou seja, os valores máximo, da média e da mediana dos teores de MO obtidos com esse último método foram maiores do que os da solução sulfocrômica (Quadro 3).

No método PMI, a quantidade de amostra analisada é medida com a pesagem deste e o teor de MO é expresso em base de massa de solo (\% p/p), enquanto no método da solução sulfocrômica a alíquota analisada é medida em volume e o resultado expresso em base de volume de solo (\% p/v). Assim, os maiores teores de MO obtidos com o método PMI podem estar relacionados também com a densidade das alíquotas pesadas, a qual diferiu entre as amostras (resultados não apresentados). Se o método PMI for utilizado em laboratórios de rotina, a forma adequada para expressar os teores de MO seria aquela cujos valores fossem o mais semelhante possível aos obtidos com o método da solução sulfocrômica, pois isso facilitaria a interpretação dos resultados do laudo de análise pelos técnicos de extensão rural. Para avaliar se a densidade das alíquotas pesadas teve influência na diferença entre os teores de $\mathrm{MO}$ obtidos com esses dois métodos, os resultados do método PMI foram expressos em base

Quadro 3. Estimativa dos teores de matéria orgânica (MO) obtidos com os métodos Walkley-Black, perda de massa por ignição e solução sulfocrômica, em amostras de solos do Rio Grande do Sul

Estimativa Walkley-Black Perda de massa por ignição Solução sulfocrômica

\begin{tabular}{|c|c|c|c|c|c|}
\hline & \multicolumn{5}{|c|}{$\mathrm{MO}, \%$} \\
\hline & \multicolumn{5}{|c|}{ Diversas unidades de mapeamento } \\
\hline & $\mathrm{m} \mathrm{m}^{-1}$ & $\mathrm{~m} \mathrm{v} \mathrm{v}^{-1}$ & $\mathrm{~m} \mathrm{~m}^{-1}$ & $\mathrm{~m} \mathrm{v}^{-1}$ & $\mathrm{~m} \mathrm{v}^{-1}$ \\
\hline Mínimo & 0,45 & 0,51 & 0,64 & 0,78 & 0,80 \\
\hline Máximo & 4,72 & 3,82 & 7,65 & 6,19 & 3,55 \\
\hline Média & 1,93 & 1,84 & 2,86 & 2,69 & 1,39 \\
\hline \multirow[t]{3}{*}{ Mediana } & 1,88 & 1,83 & 2,54 & 2,49 & 1,25 \\
\hline & \multicolumn{5}{|c|}{ Diversas unidades de mapeamento e Depressão Central } \\
\hline & $\mathrm{m} \mathrm{m}^{-1}$ & $\mathrm{~m} \mathrm{v} \mathrm{v}^{-1}$ & $\mathrm{~m} \mathrm{~m}^{-1}$ & $\mathrm{~m} \mathrm{v} \mathrm{v}^{-1}$ & $\mathrm{~m} \mathrm{v}^{-1}$ \\
\hline Mínimo & 0,37 & 0,46 & 0,60 & 0,70 & 0,43 \\
\hline Máximo & 6,31 & 5,50 & 7,65 & 6,38 & 6,08 \\
\hline Média & 2,60 & 2,53 & 3,32 & 3,22 & 2,65 \\
\hline Mediana & 2,17 & 2,11 & 3,50 & 3,22 & 2,17 \\
\hline
\end{tabular}


de massa (p/v) e de volume (p/p) e relacionados com os do método da solução sulfocrômica (p/v). Na regressão ajustada entre esses dois métodos, os valores da abscissa e da ordenada do coeficiente $b$ (intercepto) foram definidos como sendo iguais a zero (Figura 3), pois isso possibilita perceber e quantificar, facilmente, eventuais diferenças na estimativa dos teores de MO quando os resultados do método PMI são expressos $\mathrm{em} \mathrm{p} / \mathrm{p}$ e $\mathrm{p} / \mathrm{v}$.

Como observado, as regressões obtidas com os resultados dos dois conjuntos de amostras mostraram que os teores de MO observados no método PMI superestimaram os teores determinados com o método da solução sulfocrômica (Figuras 1 e 2); isso ocorreu quando os resultados do método PMI foram expressos tanto em base de p/p como em base de p/v (Figura 3). Quando expressos em base de p/p, os resultados do método PMI superestimaram em $18 \%\left(\mathrm{MO}_{\text {ROLAS }}=\right.$ $0,82 \mathrm{MO}_{\mathrm{PMI}}$; Figura $3 \mathrm{a}$ ) os teores de $\mathrm{MO}$ determinados com o método da solução sulfocrômica. Quando expressos em base de p/v, os resultados do método PMI foram $16 \%$ maiores do que os obtidos com a solução sulfocrômica $\left(\mathrm{MO}_{\mathrm{ROLAS}}=0,84 \mathrm{MO}_{\mathrm{PMI}}\right.$; Figura $\left.3 \mathrm{~b}\right)$.

Os resultados apresentados na figura 3 também indicam que o valor de $\mathrm{r}^{2}$ calculado com os teores de

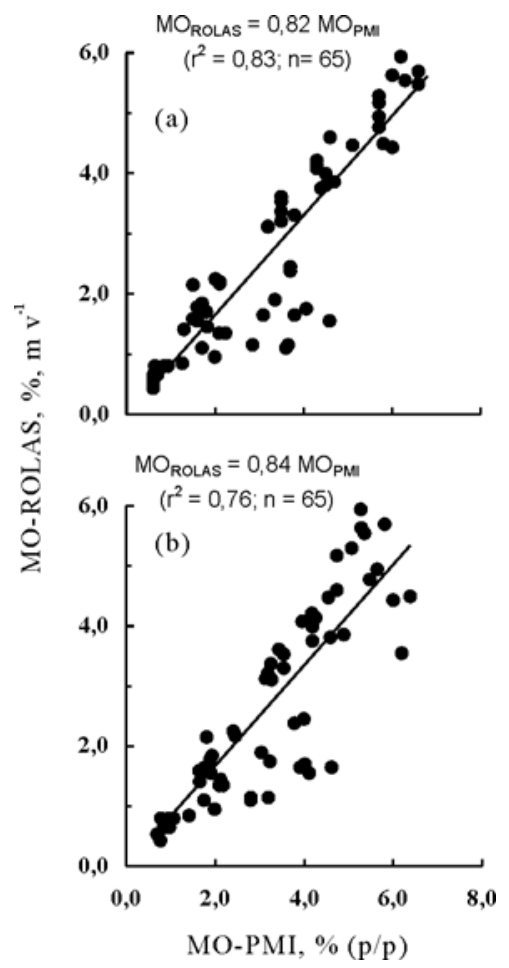

Figura 3. Relação entre os teores de matéria orgânica (MO) determinados com os métodos solução sulfocrômica (ROLAS) e perda de massa por ignição (PMI), em amostras de solos do Rio Grande do Sul. Valores obtidos com o método PMI e expressos (a) em base de massa $\left(\mathrm{m} \mathrm{m}^{-1}\right)$ e (b) em base de volume $\left(\mathrm{m} \mathrm{v}^{-1}\right)$.
MO expressos em $\mathrm{p} / \mathrm{p}\left(\mathrm{r}^{2}=0,83\right)$ foi maior do que o obtido com os resultados expressos em $\mathrm{p} / \mathrm{v}\left(\mathrm{r}^{2}=0,76\right)$. Embora a forma de expressão dos resultados obtidos com o método PMI pouco tenha influenciado a superestimativa do teor de $\mathrm{MO}$, o maior $\mathrm{r}^{2}$ obtido quando os resultados foram expressos em $\mathrm{p} / \mathrm{p}$ (Figura 3), juntamente com o fato de prescindir da determinação da densidade das alíquotas analisadas, ao contrário do observado quando expresso em base de volume, indica que a expressão dos resultados em $\mathrm{p} / \mathrm{p}$ é preferível em relação a p/v.

\section{CONCLUSÃO}

O método da perda de massa por ignição pode ser usado na análise dos teores de MO das amostras de solos usadas neste trabalho, em substituição ao método que utiliza a solução sulfocrômica.

\section{AGRADECIMENTOS}

Os autores agradecem aos Laboratórios de Solos da UPF e da UFSM, a Arnaldo Rodella e Sírio Wiethölter e à Secretaria de Ciência e Tecnologia do Rio Grande do Sul.

\section{LITERATURA CITADA}

ASSOCIAÇÃO BRASILEIRA DE NORMAS TÉCNICAS ABNT.. NBR 10.004, NBR 10.005, NBR 10.006, NBR 10.007 - Resíduos Sólidos: Coletânea de normas. 2.ed. Rio de Janeiro, 2004. 71p.

BEN-DOR, E. \& BANIN, A. Determination of organic matter content in arid-zone soils using a simple "loss-on-ignition" method. Comm. Soil Sci. Plant Anal., 20:1675-1695, 1989.

BRASIL. Ministério da Agricultura. Departamento de Pesquisa Agropecuária. Divisão de Pesquisa Pedológica. Levantamento de reconhecimento dos solos do estado do Rio Grande do Sul. Recife, DNPA, 1973. 431p. (Boletim Técnico, 30)

CAMBARDELLA, C.A.; GAJDA, A.M.; DORAN, J.W.; WIENHOLD, B.J. \& KETTLER, T.A. Estimation of particulate and total organic matter by weight loss-onignition. In: LAL, R.; KIMBLE, J.M.; FOLLET, R.F. \& STEWART, B.A., eds. Assessment methods for soil carbon. Advances in Soil Science. Boca Raton, CRC Press, 2001. p.349-359.

COMBS, S.M. \& NATHAN, M.V. Soil organic matter. In: BROWN, J.R., ed. North Central Regional Res. Publ. No. 221 (revised). Recommended chemical soil test procedures for the North Central Region. Columbia, Missouri Agric. Exp. Sta. SB 1001, 1998. p.53-58. 
COMISSÃO INTERNA DE SEGURANÇA QUÍMICA. UNIVERSIDADE ESTADUAL PAULISTA JÚLIO DE MESQUITA FILHO. INSTITUTO DE BIOCIÊNCIAS, LETRAS E CIÊNCIAS EXATAS. Os riscos do cromo (VI) e da solução sulfocrômica. Rio Preto, 2004. Disponível em: <http://www.qca.ibilce.unesp.br/prevencao/ BMCISQ1004.html>. Acesso em: 10 nov. 2005. (Boletim Mensal da Comissão Interna de Segurança Química, 10)

ESCOSTEGUY, P.A.V.; GALLIASSI, K. \& BALDISSERA, I.T. Estimativa do teor de matéria orgânica dos solos do Oeste de Santa Catarina utilizando o método da perda de peso por ignição. In: REUNIÃO ANUAL DA ROLAS, 34., Passo Fundo, 2002. Anais. Passo Fundo, Sociedade Brasileira de Ciência do Solo. Núcleo Regional Sul. ROLAS, 2003. Anexo 2. p17-25.

Escosteguy, P.A.V.; RODELlA, A.; CERETTA, C.A.; GONÇALEZ, R.; GALLIASSI, K. \& CALDEIRA, M.H. Determinação de carbono orgânico do solo utilizando o método da perda de peso por ignição. In: REUNIÃO ANUAL DA ROLAS, 33., Frederico Westphalen, 2001. Anais. Passo Fundo, Sociedade Brasileira de Ciência do Solo. Núcleo Regional Sul. ROLAS, 2002. Anexo 3. p12-19.

KIMBLE, J.M.; LAL, R. \& FOLLETT, R.F. Methods for assessing soil C pools. In: LAL, R.; KIMBLE, J.M.; FOLLET, R.F. \& STEWART, B.A., eds. Assessment methods for soil carbon. Advances in soil science. Boca Raton, CRC Press, 2001. p.3-12.
KONEN, M.E.; JACOBS, P.M.; BURRAS, C.L.; TALAGA, B.J. \& MASON, J.A. Equations for predicting soil organic carbon using loss-on-ignition for North Central U.S. Soils. Soil Sci. Soc. Am. J., 66:1878-1881, 2002.

NELSON, D.W. \& SOMMERS, L.E. Total carbon, organic carbon, and organic matter. In: SPARKS, D.L.; PAGE, A.L.; HELMKE, P.A.; LOEPPERT, R.H.; SOLTANPOUR, P.N.; TABATABAI, M.A.; JOHNSTON, C.T. \& SUMMER, M.E., eds. Methods of soil analysis. Part 3. Chemical Methods. 2.ed. Madison, SSSA-ASA, 1996. p.961-1010.

SCHULTE, E.E \& HOPKINS, B.G. Estimation of soil organic matter by weight loss-on-ignition. In: MAGDOFF, F.R.; TABATABAI, M.A. \& HANLON Jr., E.A., eds. Soil organic matter: Analysis and interpretation. Madison, SSSAASA, 1996. p.21-31.

SOCIEDADE BRASILEIRA DE CIÊNCIA DO SOLO. COMISSÃO DE QUÍMICA E FERTILIDADE DO SOLO RS/SC. Manual de adubação e calagem para os Estados do Rio Grande do Sul e de Santa Catarina. 10.ed. Porto Alegre, Sociedade Brasileira de Ciência do Solo. Núcleo Regional Sul, 2004. 400p.

TEDESCO, M.J.; GIANELLO, C.; BISSANI, C.A.; BOHNEN, H. \& VOLKWEISS, S.J. Análises de solo, plantas e outros materiais. 2.ed. Porto Alegre, Universidade Federal do Rio Grande do Sul, 1995. 174p. (Boletim Técnico, 5) 